\title{
FULLY INDECOMPOSABLE EXPONENTS OF PRIMITIVE MATRICES
}

\author{
RICHARD A. BRUALDI AND BOLIAN LIU
}

(Communicated by Thomas $\mathrm{H}$. Brylawski)

\begin{abstract}
If $A$ is a primitive matrix, then there is a smallest power of $A$ (its fully indecomposable exponent) that is fully indecomposable, and a smallest power of $A$ (its strict fully indecomposable exponent) starting from which all powers are fully indecomposable. We obtain bounds on these two exponents.
\end{abstract}

\section{INTRODUCTION}

Let $B_{n}$ denote the set of all matrices of order $n$ over the Boolean algebra $\{0,1\}$ where, in particular, $1+1=1$. Then $B_{n}$ is a semigroup whose binary operation is ordinary matrix multiplication. Let $J_{n}$ denote the matrix in $B_{n}$ each of whose entries equals 1 . A matrix $A \in B_{n}$ is primitive provided there is a positive integer $k$ such that $A^{k}=J_{n}$; the least positive integer $k$ satisfying $A^{k}=J_{n}$ is the exponent $e(A)$ of $A$. Exponents of primitive matrices have been well studied, and those numbers that are exponents of primitive matrices of order $n$ have been completely determined [4, 6, 7]. In particular the largest exponent of a primitive matrix of order $n$ is $e_{n}=n^{2}-2 n+2$. We denote the set of primitive matrices in $B_{n}$ by $P_{n}$.

A matrix $A \in B_{n}$ is partly decomposable provided for some positive integers $r$ and $s$ with $r+s=n, A$ has an $r$ by $s$ zero submatrix. A matrix in $B_{n}$ that is not partly decomposable is called fully indecomposable. It is well known that a fully indecomposable matrix is primitive and the exponents of fully indecomposable matrices have been studied [3]. We denote the set of fully indecomposable matrices in $B_{n}$ by $F_{n}$. Thus $F_{n} \subseteq P_{n}$. Moreover, it follows that

$$
P_{n}=\left\{A: A \in B_{n} \text { and } A^{k} \in F_{n} \text { for some positive integer } k\right\} .
$$

Received by the editors March 15, 1989.

1991 Mathematics Subject Classification. Primary 05C20, 05C50, 15 A48.

The first author's research was partially supported by National Science Foundation Grant No. DMS-8521521.

The second author's research was performed while a Visiting Scholar of the University of Wisconsin, Madison. 
There is a one-to-one correspondence between the set $B_{n}$ and the set $\Gamma_{n}$ of digraphs with vertex set $\{1, \ldots, n\}$. This correspondence is given as follows: If $A=\left[a_{i j}\right] \in B_{n}$, then $\Gamma(A)$ is the digraph in which there is an arc $(i, j)$ from $i$ to $j$ if and only if $a_{i j}=1(i, j=1, \ldots, n)$. We note that since $A$ may have 1 's on its main diagonal, the digraph $\Gamma(A)$ may have loops. We call a vertex $i$ of a digraph a loop-vertex provided $(i, i)$ is a loop of the digraph. We call a digraph in $\Gamma_{n}$ a primitive digraph provided it corresponds to a primitive matrix. The following properties are direct consequences of the definitions:

If $A$ is primitive, $\Gamma(A)$ is strongly connected (that is, for each pair of distinct vertices $i$ and $j$, there is a walk from $i$ to $j$ );

$A$ is primitive if and only if there is an integer $k$ such that for each pair of distinct vertices $i$ and $j$ there is a walk in $\Gamma(A)$ of length $k$ from $i$ to $j$;

[1] $A^{k}$ is fully indecomposable if and only if for each set $X$ of $r$ vertices with $0<r<n$, there are at least $r+1$ different vertices that can be reached by a walk of length $k$ which starts at a vertex in $X$.

In addition the following is a well-known characterization of primitive matrices:

$A$ is primitive if and only if the greatest common divisor of the lengths of all (elementary) cycles of $\Gamma(A)$ is 1 .

Let $A$ be a primitive matrix. It follows from (1.1) that there is a smallest positive integer $k$ such that $A^{k}$ is fully indecomposable; we denote the smallest such integer by $f(A)$. Schwarz [5] raised the question of determining the numbers

$$
f_{n}=\max \left\{f(A): A \in P_{n}\right\} \quad(n \geq 1) .
$$

Before proceeding we observe an important difference that occurs in the investigation of the numbers $e(A)$ and $f(A)$ for primitive matrices $A$. If $A^{k}=J_{n}$, then because $A$ can have no zero rows, $A^{i}=J_{n}$ for all $i \geq k$. However, if $A^{k} \in F_{n}$, then it does not necessarily follow that $A^{i} \in F_{n}$ for all $i \geq k$. For example, let

$$
A=\left[\begin{array}{lllllll}
0 & 1 & 0 & 0 & 0 & 0 & 0 \\
0 & 0 & 1 & 0 & 0 & 0 & 0 \\
0 & 0 & 0 & 1 & 0 & 1 & 1 \\
0 & 0 & 0 & 0 & 1 & 0 & 0 \\
1 & 0 & 0 & 0 & 0 & 0 & 0 \\
1 & 0 & 0 & 0 & 0 & 0 & 0 \\
1 & 0 & 0 & 0 & 0 & 0 & 0
\end{array}\right] .
$$

Then $A^{i} \notin F_{7}(i=1, \ldots, 7) ; A^{8}, A^{9} \in F_{7} ; A^{10}, A^{11} \notin F_{7}$; and $A^{i} \in F_{7}$ $(i \geq 12$ ). (It follows (cf. (1.1)) that $A$ is primitive since some power of $A$ is 
fully indecomposable.) For a primitive matrix $A$ we define $f^{*}(A)$ to be the smallest positive integer $k$ such that $A^{i}$ is fully indecomposable for all $i \geq k$. We call $f(A)$ and $f^{*}(A)$, respectively, the fully indecomposable exponent and strict fully indecomposable exponent of the primitive matrix $A$. The matrix $A$ in (1.6) satisfies $f(A)=8$ and $f^{*}(A)=12$. In general we have

$$
f(A) \leq f^{*}(A) \leq e(A) \quad\left(A \in P_{n}\right) .
$$

We define

$$
f_{n}^{*}=\max \left\{f^{*}(A): A \in P_{n}\right\} \quad(n \geq 1),
$$

and hereby raise the question of determining the numbers $f_{n}^{*}$. Clearly $f_{n} \leq$ $f_{n}^{*}(n \geq 1)$.

In what follows we obtain bounds for the numbers $f_{n}$ and $f_{n}^{*}$.

\section{BOUNDS ON THE EXPONENTS}

Let $A$ be a matrix in $B_{n}$ with associated digraph $\Gamma(A)$, and let $k$ be a nonnegative integer. For $X \subseteq\{1, \ldots, n\}, R_{k}(X)$ denotes the set of all those vertices that can be reached by a walk of length $k$ in $\Gamma(A)$ starting from a vertex in $X$. (If $k=0, R_{k}(X)=X$.) A restatement of (1.4) is:

$$
\begin{aligned}
& A^{k} \text { is fully indecomposable if and only if }\left|R_{k}(X)\right|>|X| \text { for all } \\
& X \text { with } \phi \neq X \varsubsetneqq\{1, \ldots, n\} \text {. }
\end{aligned}
$$

Chao and Zhang [2] showed that $f(A) \leq n$ if $A \in P_{n}$ and $\operatorname{trace}(A) \neq 0$. We refine this result to obtain an inequality for $f^{*}(A)$ in Theorem 2.2.

Lemma 2.1. Let $\Gamma$ be a strongly connected digraph with vertex set $\{1, \ldots, n\}$, let $s$ be a positive integer, and let $Z=\left\{i_{1}, \ldots, i_{s}\right\}$ be a set of $s$ loop-vertices of $\Gamma$. Then for each positive integer $t$,

$$
\left|R_{t}(Z)\right| \geq \min \{s+t, n\} \text {. }
$$

Proof. Suppose that $R_{t}(Z) \neq\{1, \ldots, n\}$. Since $\Gamma$ is strongly connected, there is an $\operatorname{arc}(p, j)$ from some vertex $p \in R_{t}(Z)$ to a vertex $j \notin R_{t}(Z)$. Because $j \notin R_{t}(Z)$ there is a vertex in $Z$, say $i_{1}$, such that the distance from $i_{1}$ to $p$ is $t$ and the distance from each of $i_{2}, \ldots, i_{s}$ to $p$ is at least $t$. Thus there is a walk $\left(i_{1}, \ldots, p\right)$ of length $t$ from $i_{1}$ to $p$ containing $t+1$ distinct vertices all of which are different from $i_{2}, \ldots, i_{s}$. Since $i_{1}, i_{2}, \ldots, i_{s}$ are loop-vertices, we conclude that

$$
\left|R_{t}(Z)\right| \geq(s-1)+(t+1)=s+t,
$$

and the lemma follows.

Theorem 2.2. Let $s$ be a positive integer, and let $A$ be a matrix in $P_{n}$ having $s$ 1's on its main diagonal. Then

$$
f^{*}(A) \leq n-s+1
$$


Proof. Let $Z$ be the set of $s$ loop-vertices of the digraph $\Gamma(A)$, and let $t$ be a positive integer. Let $X$ be a set of vertices with $\phi \neq X \varsubsetneqq\{1, \ldots, n\}$, and let $k=|X|$. We show that

$$
\left|R_{t}(X)\right| \geq|X|+1 \text { for } t \geq n-s+1 \text {. }
$$

If $\left|R_{t}(X)\right|=n$, then (2.4) holds. We now suppose that $\left|R_{t}(X)\right|<n$. First assume that $X \cap Z \neq \varnothing$. By Lemma 2.1

$$
\left|R_{t}(X)\right| \geq\left|R_{t}(X \cap Z)\right| \geq|X \cap Z|+t .
$$

Hence if $t \geq n-s+1$,

$$
\left|R_{t}(X)\right| \geq|X \cap Z|+n-s+1 \geq|X|+1 \text {. }
$$

Now assume that $X \cap Z=\varnothing$. Let $x^{*}$ be a vertex in $X$ and let $z^{*}$ be a vertex in $Z$ such that $x^{*}$ has the minimum distance $d$ to $z^{*}$ among all vertices $x$ in $X$ and $z$ in $Z$. Then

$$
d \leq n+1-|Z|-|X|=n+1-s-k .
$$

Because $z^{*}$ is a loop-vertex, there are walks of length $t$ from $x^{*}$ to each vertex in $R_{k}\left(z^{*}\right)$ for each integer $t \geq(n+1-s-k)+k=n+1-s$. Using Lemma 2.1 we obtain that

$$
\left|R_{t}(X)\right| \geq\left|R_{t}\left(\left\{x^{*}\right\}\right)\right| \geq\left|R_{k}\left(z^{*}\right)\right| \geq k+1
$$

for each integer $t \geq n+1-s$. Hence (2.4) holds, and using (1.4) we conclude that (2.3) holds.

It is easy to construct matrices $A$ satisfying the hypotheses of Theorem 2.2 for which equality holds in (2.3). Let $s$ and $n$ be positive integers with $s \leq n$, and let $\Gamma$ be the digraph obtained by including a loop at each of $s$ consecutive vertices of a simple cycle of length $n$. Then the matrix $A \in B_{n}$ whose associated digraph is $\Gamma$ is in $P_{n}$ and satisfies $f^{*}(A)=n+1-s$.

Corollary 2.3 (cf. [2]). Let the matrix $A$ in $P_{n}$ have nonzero trace. Then

$$
f(A) \leq f^{*}(A) \leq n .
$$

Corollary 2.4. Let $A$ be a matrix in $P_{n}$. Suppose that the digraph $\Gamma(A)$ has a cycle of length $r$ and that there are $s$ vertices which belong to at least one cycle of length $r$. Then

$$
f(A) \leq r(n-s+1) .
$$

Proof. The matrix $A^{r}$ has $s$ 1's on its main diagonal, and hence by Theorem $2.2\left(A^{r}\right)^{n-s+1}$ is fully indecomposable.

The matrix

$$
A=\left[\begin{array}{llll}
0 & 1 & 0 & 1 \\
0 & 0 & 0 & 1 \\
0 & 1 & 0 & 0 \\
1 & 0 & 1 & 0
\end{array}\right]
$$


satisfies the hypotheses of Corollary 2.4 with $r=3$ and $s=4$. Hence by (2.6), $f(A) \leq 3$. Since $A^{2} \neq J_{4}, f(A)=3$ and equality holds in (2.6).

We note the following consequences of Corollary 2.4:

$$
\text { If } \Gamma(A) \text { has a Hamilton cycle (so } r=s=n \text { ), then } f(A) \leq n \text {. }
$$

If $A$ is symmetric (so $r=2$ and $s=n$ ), then $f(A) \leq 2$.

Corollary 2.5. Let $A$ be a matrix in $P_{n}$. Suppose that the digraph $\Gamma(A)$ has diameter $d$. Then $f(A) \leq 2 d(n-d)$.

Proof. Since $\Gamma(A)$ is strongly connected, there is a cycle of length $r \leq 2 d$ containing $s \geq d+1$ distinct vertices. Hence by (2.6), $f(A) \leq 2 d(n-d)$.

We now use Corollary 2.4 to obtain an upper bound on the numbers $f_{n}(n \geq 1)$.

Theorem 2.6. $f_{n} \leq\left\lceil\frac{1}{4}(n-1)(n+3)\right\rceil$ for $n \geq 1$.

Proof. Let $A$ be a matrix in $P_{n}$. Since $\Gamma(A)$ is strongly connected, $\Gamma(A)$ has a simple cycle of length $r$ for some $r$ with $1 \leq r \leq n$. Let $s$ be the number of vertices which belong to at least one cycle of length $r$. Then $s \geq r$ and by Corollary 2.4 ,

$$
f(A) \leq r(n-s+1) \leq r(n-r+1) .
$$

For integral $r, r(n-r+1)$ achieves its maximum when $r=(n+1) / 2$ ( $n$ odd) and $r=n / 2, n / 2+1$ ( $n$ even). Since $A$ is primitive, then by (1.5) the greatest common divisor of the lengths of the cycles is 1 . Hence if $n$ is odd and $r=(n+1) / 2, \Gamma(A)$ has a cycle of length different from $(n+1) / 2$. We now obtain from (2.6) that

$$
f(A) \leq \begin{cases}\left(n^{2}+2 n\right) / 4, & (n \text { even }) \\ \left(n^{2}+2 n-3\right) / 4, & (n \text { odd })\end{cases}
$$

and the theorem follows.

The upper bound for $f_{n}$ in Theorem 2.6 probably is not of the same order of magnitude as $f_{n}$. The example (1.6) can be generalized to all $n \geq 5$ to show that $f_{n} \geq 2 n-4$. Indeed it is tempting to conjecture that $f_{n}=2 n-4 \quad(n \geq 5)$.

We now consider the maximum strict fully indecomposable exponent $f_{n}^{*}$. First we obtain by example a lower bound for $f_{n}^{*}$.

Suppose that $k$ and $n$ are integers with $n \geq 5$ and $2 \leq k \leq n-3$. Let $A$ be the matrix in $B_{n}$ whose associated digraph is pictured in Figure 1 (p. 1198). This digraph has cycles of lengths $n-k+1$ and $n-k$, and hence by (1.5) $A \in P_{n}$. Let $X_{k}=\{n-k+1, \ldots, n\}$. One easily checks that

$$
\left|R_{i(n-k)-1}\left(X_{k}\right)\right|=i \quad(i=1, \ldots, k) .
$$

Hence it follows from (2.1) that

$$
f^{*}(A) \geq k(n-k)
$$




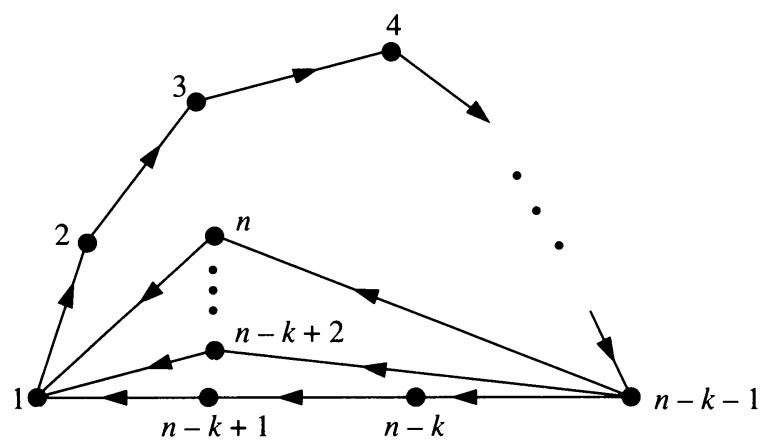

FigURE 1. A digraph.

Indeed one may show that $f^{*}(A)=k(n-k)$. Taking $k=\lfloor n / 2\rfloor$ in (2.10) we obtain

$$
f_{n}^{*} \geq\lfloor n / 2\rfloor\lceil n / 2\rceil \quad(n \geq 5) .
$$

For a primitive matrix $A$ in $B_{n}, f^{*}(A) \leq e(A) \leq e_{n}=n^{2}-2 n+2$ and hence it follows that

$$
f_{n}^{*} \leq n^{2}-2 n+2
$$

Let $\lambda(A)$ denote the number of distinct lengths of the cycles of $\Gamma(A)$. It follows from (1.5) that $\lambda(A) \geq 2$ if $n>1$. We now turn our attention to obtaining an improved bound for $f^{*}(A)$ in the case that $\lambda(A)=2$.

Lemma 2.7. Let $\Gamma$ be a digraph in $\Gamma_{n}$, and let $\gamma$ be a cycle of $\Gamma$ of length $r$. If $X$ is a set of vertices belonging to the cycle $\gamma$, then

$$
R_{i r+j}(X) \subseteq R_{(i+1) r+j}(X) \quad(i \geq 0 ; 0 \leq j \leq r-1) .
$$

Proof. If from some $x$ in $X$ there is a path of length $i r+j$ to a vertex $z$, then there is also a walk from $x$ to $z$ of length $(i+1) r+j$.

Lemma 2.8. Let $\Gamma$ be a digraph in $\Gamma_{n}$, and let $\gamma$ be a cycle of $\Gamma$ of length $r$. Let $X$ be the set of all vertices belonging to the cycle $\gamma$. Then

$$
R_{i}(X) \subseteq R_{i+1}(X) \quad(i \geq 0) .
$$

Proof. If from some vertex $x$ of $X$ there is a walk of length $i$ to a vertex $z$, then there is a walk of length $i+1$ to $z$ from the vertex which precedes $x$ in $\Gamma$.

Corollary 2.9. If $Z$ is the set of vertices of $\Gamma$ consisting of those vertices $z$ for which there is a walk of length at most $d$ to $z$ from some vertex of the cycle $\gamma$, then $R_{d}(X)=Z$.

Lemma 2.10. Let $r$ and $s$ be relatively prime positive integers with $r>s$. Let $\Gamma$ be a digraph in $\Gamma_{n}$ with exactly two cycles $\gamma$ and $\mu$ where $\gamma$ has length $r$, 


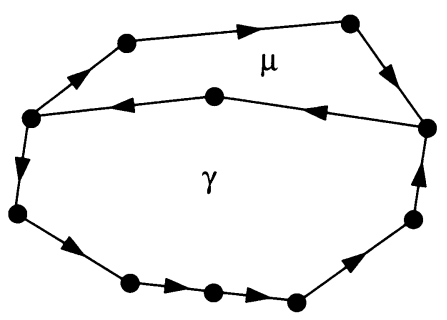

FIGURE 2. A digraph satisfying the hypotheses of Lemma 2.10 with $n=10, r=8$, and $s=5$.

$\mu$ has length $s$, and $\gamma$ and $\mu$ intersect. Let $X$ be a nonempty set of vertices of the cycle $\gamma$. Then

$$
\left|R_{i}(X)\right| \geq \min \{n,|X|+l\} \quad \text { if } i \geq l r \text { and } l \geq 1 .
$$

Proof. A digraph $\Gamma$ satisfying the hypotheses is pictured in Figure 2. Let $Z$ be the set of vertices of $\gamma$, so that $\phi \neq X \subseteq Z$. For $i \geq 1$ let $X^{(i)}$ denote the set of vertices of $\gamma$ that are reachable from $X$ by a walk in $\gamma$ of length $i$. It follows that $X^{(i)}=X^{(j)}$ if $i \equiv j(\bmod r)$. We first show that for $X \neq Z$, we have $\left|R_{r}(X) \cap Z\right|>|X|$. Since $X=X^{(r)} \subseteq R_{r}(X) \cap Z$, it suffices to show that $\left(R_{r}(X) \backslash X\right) \cap Z$ is nonempty. Suppose this set were empty. Then making use of the cycle $\mu$ we see that $X^{(r-s)} \subseteq R_{r}(X)$ and hence $X^{(r-s)}=X$. This implies that $X=X^{(s)}$ which contradicts the fact that $r$ and $s$ are relatively prime. It follows that (2.12) holds if $i=l r$ and $l \geq 1$. It now suffices to show that (2.12) holds for $l=1$ and $r+1 \leq i<2 r$.

If $X=Z$, then using Lemma 2.8 we see that

$$
\left|R_{i}(X)\right| \geq \min \{n,|X|+i\}
$$

for all $i \geq 1$. Hence we may assume that $X \neq Z$. If $R_{i}(X) \nsubseteq Z$, then the desired conclusion holds. We now assume that $R_{i}(X) \subseteq Z$. Contrary to what we wish to prove, we assume that $\left|R_{i}(X)\right|=|X|$, from which it follows that $R_{i}(X)=X^{(i)}$. Since $R_{i}(X) \subseteq Z$, we have that $R_{i-s}(X) \subseteq R_{i}(X)$. It follows that $X^{(i-s)} \subseteq X^{(i)}$ and hence that $X^{(i-s)}=X^{(i)}$, implying that the set $Y=X^{(i-s)}$ of vertices of $\gamma$ satisfies $Y^{(s)}=Y$. Again we contradict the assumption that $r$ and $s$ are relatively prime. The proof of the lemma is now complete.

We now obtain a bound for the strict fully indecomposable exponent $f^{*}(A)$ of a primitive matrix $A$ for which the number $\lambda(A)$ of distinct cycle lengths of the digraph $\Gamma(A)$ equals 2 .

Theorem 2.11. Let $A$ be a matrix in $P_{n}$ satisfying $\lambda(A)=2$. Then

$$
f^{*}(A) \leq\left\lfloor(n+1)^{2} / 4\right\rfloor \text {. }
$$


Proof. Let the lengths of the cycles of $\Gamma(A)$ be $r$ and $s$ where $r>s$, and $r$ and $s$ are relatively prime. There exist cycles $\gamma$ and $\mu$ of $\Gamma$ with lengths $r$ and $s$, respectively, such tht $\gamma$ and $\mu$ intersect. Let the digraph $\Gamma^{*}$ consist of the vertices and arcs of $\gamma$ and $\mu$, and let $m$ be the number of vertices of $\Gamma^{*}$. Let $Y$ be a subset of the vertices of $\Gamma$ with $1 \leq k=|Y| \leq n-1$. First suppose that $\gamma$ and $\mu$ above can be chosen so that $Y$ contains $p \geq 1$ vertices of $\gamma$. Applying Lemma 2.10 and Corollary 2.9, we see that

$$
\left|R_{i}(Y)\right| \geq k+1 \quad(i \geq(k-p+1) r) .
$$

Since $r \leq n-(k-p)$, an easy calculation shows that

$$
(k-p+1) r \leq\left\lfloor\frac{1}{4}(n+1)^{2}\right\rfloor .
$$

Now suppose that $\gamma$ and $\mu$ cannot be chosen so that $\gamma$ contains a vertex of $Y$. In this case $r \leq n-k$. There is a walk of length $t$ from some vertex in $Y$ to some vertex $x$ of $\gamma$ where $t \leq n-r-k+1$. Applying Lemma 2.10 and Corollary 2.9 again, we see that

$$
\left|R_{i}(\{x\})\right| \geq k+1 \quad(i \geq k r) .
$$

Hence

$$
\left|R_{i}(Y)\right| \geq k+1 \quad(i \geq k r+n-r-k+1) .
$$

An easy calculation now shows that

$$
k r+n-r-k+1 \leq\left\lfloor n^{2} / 4\right\rfloor+1 .
$$

It follows that for all $Y$ with $\phi \neq Y \varsubsetneqq X$,

$$
\left|R_{i}(Y)\right| \geq|Y|+1 \quad\left(i \geq\left\lfloor(n+1)^{2} / 4\right\rfloor\right) .
$$

Hence $A^{i}$ is fully indecomposable for all integers $i \geq\left\lfloor(n+1)^{2} / 4\right\rfloor$.

We conclude with the following remark. Let $A$ be a primitive matrix of order $n$. The exponent $e(A)$ of $A$ satisfies $e(A) \leq n^{2}-2 n+2$. If $\lambda(A) \geq 3$, then by a theorem of Lewin and Vitek [4], $e(A) \leq\left\lfloor\left(n^{2}-2 n+2\right) / 2\right\rfloor+1$. In particular, the largest exponent for matrices in $P_{n}$ occurs among those matrices with $\lambda(A)=2$. We conjecture that the strict fully indecomposable exponent behaves in a similar way and thus that

$$
f_{n}^{*} \leq\left\lfloor(n+1)^{2} / 4\right\rfloor \text {. }
$$

In view of $(2.11)$ the validity of this latter inequality would imply that $f_{n}^{*}$ is roughly $n^{2} / 4$.

\section{REFERENCES}

1. C. Y. Chao, On a conjecture of the semigroup of fully indecomposable relations, Czechoslovak Math. J. 27 (1977), 591-597.

2. C. Y. Chao and M. C. Zhang, On the semigroup of fully indecomposable relations, Czechoslovak Math. J. 33 (1983), 314-319. 
3. M. Lewin, Bounds for exponents of doubly stochastic primitive matrices, Math. Z. 137 (1974), 21-30.

4. M. Lewin and Y. Vitek, $A$ system of gaps in the exponent set of primitive matrices, Illinois J. Math. 25 (1981), 87-98.

5. S. Schwarz, The semigroup of fully indecomposable relations and Hall relations, Czechoslovak Math. J. 23 (1973), 151-163.

6. J. Y. Shao, On a conjecture about the exponent set of primitive matrices, Linear Algebra Appl. 65 (1985), 91-123.

7. K. M. Zhang, On Lewin and Vitek's conjecture about the exponent set of primitive matrices, Linear Algebra Appl. 96 (1987), 101-108.

Department of Mathematics, University of Wisconsin, Madison, Wisconsin 53706

E-mail address: brualdi@math.wisc.edu

Department of Mathematics, South China Normal University, Guangzhou, People's RePUBLIC OF CHINA 